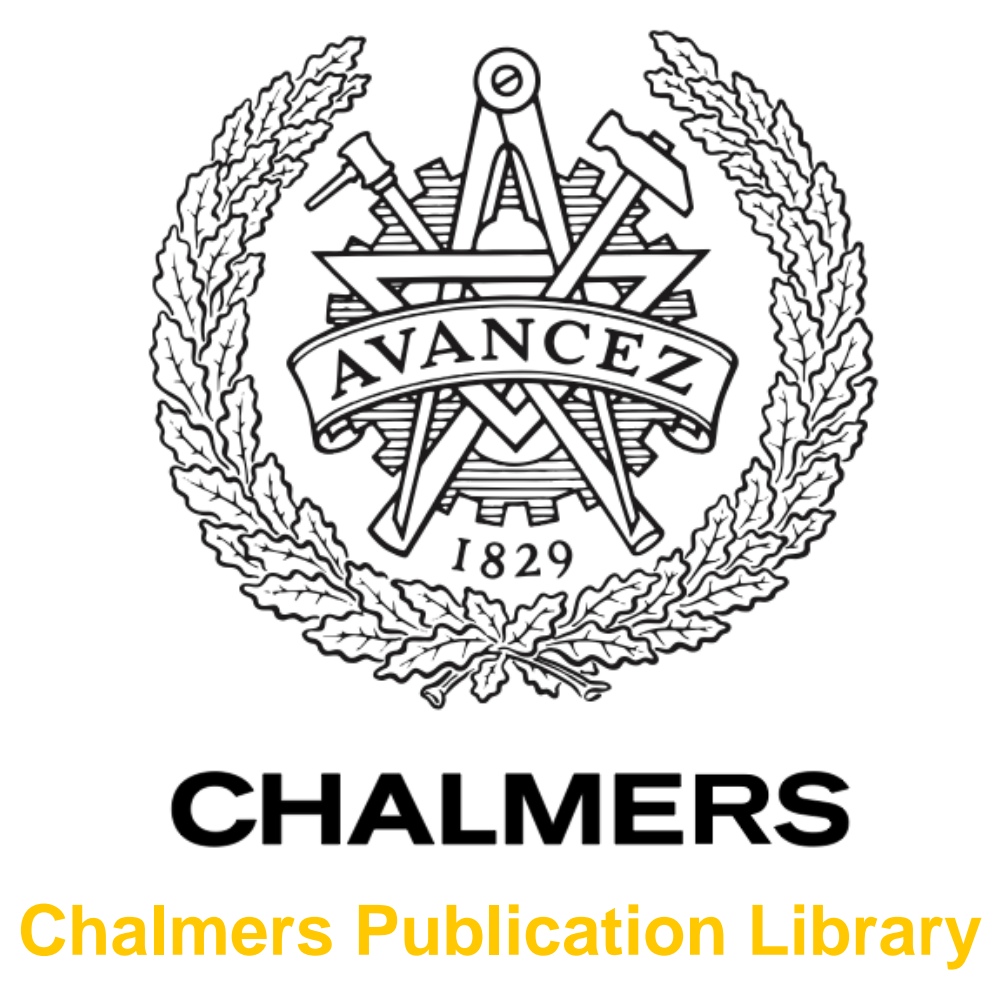

\title{
Effects of parallel ion motion on electromagnetic toroidal ion temperature gradient modes in a fluid model
}

This document has been downloaded from Chalmers Publication Library (CPL). It is the author's version of a work that was accepted for publication in:

Physics of Plasmas (ISSN: 1070-664X)

Citation for the published paper:

Jarmén, A. ; Anderson, J. ; Malinov, P. (2015) "Effects of parallel ion motion on electromagnetic toroidal ion temperature gradient modes in a fluid model". Physics of Plasmas, vol. 22(8), pp. 082508.

Downloaded from: http://publications.lib.chalmers.se/publication/220753

Notice: Changes introduced as a result of publishing processes such as copy-editing and formatting may not be reflected in this document. For a definitive version of this work, please refer to the published source. Please note that access to the published version might require a subscription. 


\section{AIP $\left.\right|_{\text {Physics of }}$

\section{Effects of parallel ion motion on electromagnetic toroidal ion temperature gradient modes in a fluid model}

A. Jarmén, J. Anderson, and P. Malinov

Citation: Physics of Plasmas 22, 082508 (2015); doi: 10.1063/1.4928374

View online: http://dx.doi.org/10.1063/1.4928374

View Table of Contents: http://scitation.aip.org/content/aip/journal/pop/22/8?ver=pdfcov

Published by the AIP Publishing

\section{Articles you may be interested in}

Kinetic water-bag model of global collisional drift waves and ion temperature gradient instabilities in cylindrical geometry

Phys. Plasmas 20, 042105 (2013); 10.1063/1.4799814

Mean sheared flow and parallel ion motion effects on zonal flow generation in ion-temperature-gradient mode turbulence

Phys. Plasmas 13, 102304 (2006); 10.1063/1.2357892

Linear gyrokinetic calculations of toroidal momentum transport in a tokamak due to the ion temperature gradient mode

Phys. Plasmas 12, 072515 (2005); 10.1063/1.1949608

Critical gradient formula for toroidal electron temperature gradient modes

Phys. Plasmas 8, 4096 (2001); 10.1063/1.1391261

Electromagnetic effects on toroidal-ion-temperature-gradient modes and associated nonthermal cross-field transports

Phys. Plasmas 7, 1132 (2000); 10.1063/1.873921

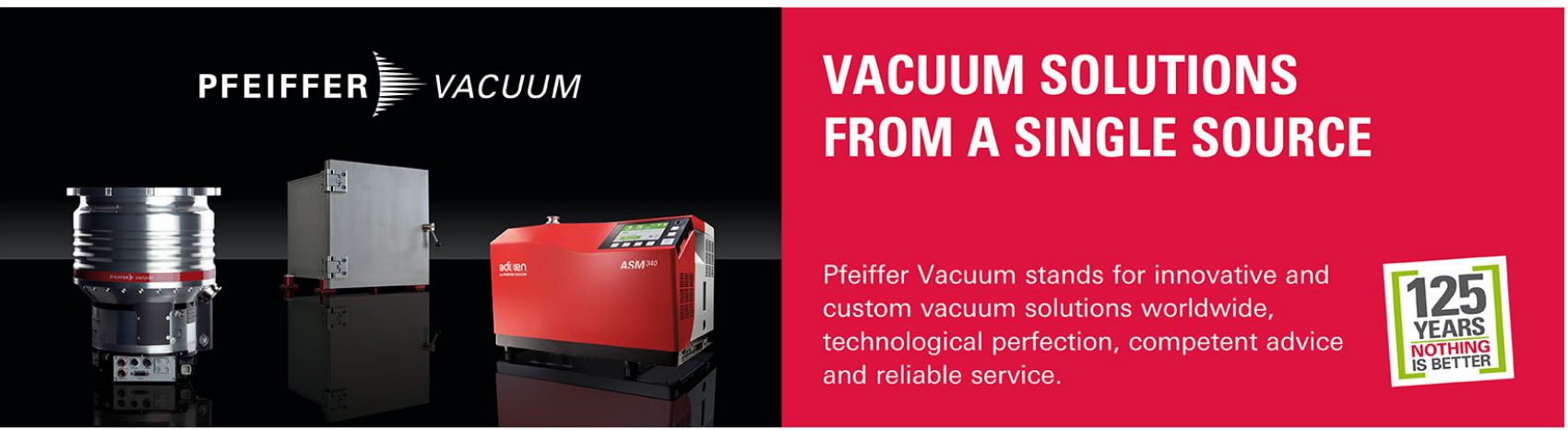




\title{
Effects of parallel ion motion on electromagnetic toroidal ion temperature gradient modes in a fluid model
}

\author{
A. Jarmén, ${ }^{1}$ J. Anderson, ${ }^{2}$ and P. Malinov ${ }^{3}$ \\ ${ }^{1}$ Chalmers University of Technology and EURATOM-VR Association, Gothenburg, Sweden \\ ${ }^{2}$ Department of Earth and Space Sciences, Chalmers University of Technology, Gothenburg, Sweden \\ ${ }^{3}$ Faculty of Physics, Sofia University, Sofia, Bulgaria
}

(Received 12 February 2015; accepted 16 July 2015; published online 14 August 2015)

\begin{abstract}
Effects of ion dynamics along the background magnetic field have been added to an advanced fluid model which has been developed, tested, and successfully used in transport code applications during the last decades. Introducing electrostatic $(\phi)$ and electromagnetic $(\psi)$ potentials, a system of two coupled second order differential equations in these potentials is derived. The mode solution is interpreted as a coupling between an Ion Temperature Gradient (ITG) mode and an ion motion driven acoustic wave. The mode may be stabilized by electromagnetic effects and by minimizing the ITG parameter $\eta_{i}\left(=\frac{L_{n}}{L_{T i}}\right)$. Interestingly, the addition of kinetic Landau resonance effects may enhance the $\eta_{i}$ stabilization. (C) 2015 AIP Publishing LLC. [http://dx.doi.org/10.1063/1.4928374]
\end{abstract}

\section{INTRODUCTION}

For the control of instabilities and transport in fusion devices, the study of low frequency perturbations is an important task. Specifically, the interest in instabilities driven by inhomogeneous temperature profiles has been strong during the last decades along with the installation of auxiliary heating equipment in a number of experimental plants.

Lately, gyrokinetic transport codes and advanced fluid modelling of drift wave turbulence have been successful in explaining experimental results. ${ }^{1-9}$ Although a kinetic description of the plasma is more detailed than a fluid description, the latter may give simpler and more explicit results. ${ }^{10-24}$ Also the access of computing power is in general not crucial with fluid codes. They normally may be run for times longer than the transport time scale contrary to kinetic codes. This is one of the reasons for the wide use of the advanced fluid transport model in Refs. 1 and 5. This is also a motive for the physics explored in this paper, although electromagnetic gyrokinetic and gyrofluid models also allow this type of investigations.

For toroidal plasmas, the applicability of fluid models has been a problem due to the magnetic curvature drift resonance at $\omega \approx \omega_{D}$, where $\omega_{D}$ is the magnetic drift frequency. This resonance has made it difficult to evaluate the kinetic integral analytically without expanding in $\frac{\omega_{D}}{\omega}$. In this paper, a fluid model for ions is used which is valid also in the regime $\omega_{D}>\omega$. A basic presentation of the model is given in Ref. 1. It includes all the curvature effects in the continuity and energy equations, and an important role is played by the ion diamagnetic heat flow which is used in the energy equation to close the fluid hierarchy. ${ }^{1,2,4,5}$ The model is basically reactive (i.e., collisionless) and has been successfully used to study effects such as electron trapping and impurities on mode structure and particle and energy transport and confinement. $^{11-13,18-25}$

In the original version of the model, the phase velocity of the drift modes along the background magnetic field is assumed to be much larger than the ion thermal velocity.
The ion dynamics in this direction may then be neglected which simplifies the analytical treatment. This is based on the assumption $|\omega|>\omega_{t i}$, where $\omega_{t i}$ is the ion transit frequency. A complication in the description of drift mode turbulence is, however, that the phase velocities of the turbulent perturbations may be comparable to the thermal velocities in the plasma. In this work, the parallel ion dynamics (parallel = along the background magnetic field) has been added in the full model (i.e., including full electromagnetic effects). Results are presented both with and without kinetic Landau resonances taken into account.

In Ref. 11, our model has been compared with an adiabatic ExB convection model and with a model where dissipative wave-particle resonances are taken into account by adding gyro-Landau contributions ${ }^{26-28}$ to the heat flow in the energy equation. Electromagnetic effects are, however, neglected in this paper. In Ref. 29, the local model (i.e., excluding parallel dynamics) with added drift kinetic resonances has been compared with a gyrokinetic model. A qualitative agreement was found. In Ref. 30, the model has also been compared with an electromagnetic local kinetic model, and in Ref. 31 the model has been used to study zonal flow generation. Also here, results from the models were in good qualitative agreement. More recently, the gyrofluid model TGLF ${ }^{32,33}$ was developed where trapped particles and short wavelength electron modes are taken into account. Concerning dissipative wave-particle resonances, an interesting question is to what extent nonlinear effects in velocity space may drive the particles away from resonance and thus decrease the motivation for adding gyro-Landau contributions in the fluid hierarchy.

The analytical treatment is shown to give two coupled differential equations in the electrostatic $(\Phi)$ and electromagnetic $(\Psi)$ potentials, each equation being of second order. In the electrostatic limit, the system is reduced to one second order equation which is also the case when parallel ion dynamics is neglected. The generalized model is used to study mode structure and stability with focus on the Ion 
Temperature Gradient (ITG) mode in toroidal (Tokamak) geometry. The ballooning mode formalism ${ }^{34}$ is applied and a shooting code has been developed, where $\Phi$ and $\Psi$ are shot simultaneously. Approximations in the (linearised) basic equations are avoided and no assumptions are made a priori about, e.g., strong ballooning $(\theta=0)$ or structure (Gauss, harmonic) of the mode in ballooning space. Kinetic waveparticle resonances are taken into account by adding Landau contributions to the heat flow according to Refs. 26-28. In this contribution, however, a semilocal mode approximation ${ }^{10,35}$ has been used in order to avoid a system of equations higher than second order.

It is shown that the parallel ion motion removes the characteristic ITG mode sharp $\beta$ stabilization ${ }^{12,13}$ ( $\beta=$ plasma pressure/magnetic field pressure) and reduces the growth rate. It is also shown that the parallel motion relaxes and shifts the sharp magnetic drift resonance in the ballooning structure of the electrostatic potential $\Phi$. The $\eta_{i}$ dependence of the mode $\left(\eta_{i}=\frac{L_{n}}{L_{T}}\right)$ shows that marginal stability is shifted upwards when ion transit effects are present. The trend is seen in Ref. 11, although finite $\beta$ effects are absent there. With our choice of parameters (similar to the Cyclone DIII-D case ${ }^{36}$ ), the acoustic mode (not being sensitive to $\eta_{i}$ ) weakens the pure $\eta_{i}$ mode. Similar to this one, it is driven by the compressibility accomplished by curvature $\left(\epsilon_{n} \propto \frac{1}{R}\right)$. As was the case for the $\beta$ dependence, we find a strong ballooning and magnetic resonance in the electrostatic potential in the absence of ion transit effects. This is relaxed to a substantially weaker ballooning and resonance when parallel dynamics is taken into account. Interestingly, the signatures of the strong ballooning and resonance do not show in the electromagnetic potential due to the basically electrostatic character of the mode. Toroidicity, i.e., the magnetic field curvature $\left(\propto \frac{1}{R}\right)$ inherent in the $\epsilon_{n}$ parameter $\left(\epsilon_{n}=\frac{2 L_{n}}{R}\right)$, stabilizes the pure $\eta_{i}$ mode at large values. With parallel ion dynamics, the stabilization is enhanced and the growth rate is reduced by influence of the acoustic mode. Accounting for kinetic Landau resonances in the model interestingly enhances the $\eta_{i}$ stabilization but does not significantly change the results of the $\beta$ and $\epsilon_{n}$ dependencies.

Altogether, it is shown that the interaction between the ion acoustic and ITG modes enables modes with reduced (real) frequencies and growth rates and which depend on the magnetic curvature $\left(\propto \frac{1}{R}\right)$.

The paper starts with a derivation of the two coupled differential equations in $\Phi$ and $\Psi$. It is followed by a presentation of numerical results where comparisons with the case without parallel ion dynamics are made. Dependencies of the eigenfrequency $(\omega)$ on the electromagnetic $(\beta)$, ITG $\left(\eta_{i}\right)$, and toroidicity $\left(\epsilon_{n}\right)$ parameters are shown together with the mode structure in the ballooning mode representation. A summary finally concludes the paper.

\section{FORMULATION}

We consider a tokamak equilibrium with Shafranov shifted circular magnetic surfaces and a perturbation of the form $\exp \left(j \mathbf{k}_{\perp} \cdot \mathbf{x}_{\perp}+j \mathbf{k}_{\|} \cdot \mathbf{x}_{\|}-j \omega t\right)$, where $\mathbf{k}_{\|}$and $\mathbf{k}_{\perp}$ are directed parallel and perpendicular to the background magnetic field $\mathbf{B}_{0}$, respectively. Omitting the parallel magnetic perturbation (the compressional Alfvén mode), we adopt the following representation for the perturbed electric and magnetic fields:

$$
\begin{aligned}
& \mathbf{E}=-\nabla \phi-\frac{\partial \mathbf{A}_{\|}}{\partial t}, \\
& \mathbf{B}=\mathbf{B}_{\perp}=\nabla \times \mathbf{A}_{\|},
\end{aligned}
$$

where $\phi$ is the scalar potential, and $\mathbf{A}_{\|}$is the parallel vector potential.

\section{A. Electron model}

From the parallel momentum equation for the electrons neglecting electron inertia, we obtain

$$
k_{\|} \frac{\delta n_{e}}{n_{0}}=\left[k_{\|} \phi-\left(\omega-\omega_{* e}\right) A_{\|}\right] \frac{e}{T_{e}},
$$

where $\delta n_{e}$ is the electron density perturbation, $\omega_{* e}=\mathbf{k}_{\perp} \cdot \mathbf{v}_{* e}$ is the electron diamagnetic frequency, and $\mathbf{v}_{* e}=-T_{e} \frac{e_{\|} \times \nabla n_{0}}{e n_{0} B_{0}}$ the electron diamagnetic drift velocity (with only density gradient included). Assuming isothermal electrons, i.e., a large parallel electron heat conductivity, we have $\nabla_{\|} T_{e}=0$, where $\nabla_{\|}$is taken along the total magnetic field (i.e., background plus perturbed field). This gives the electron temperature perturbation

$$
k_{\|} \frac{\delta T_{e}}{T_{e}}=\eta_{e} \omega_{* e} \frac{e A_{\|}}{T_{e}},
$$

where $\eta_{e}=\frac{L_{n_{e}}}{L_{T_{e}}}$ is the ratio between the characteristic scale lengths of density and electron temperature. From the electron continuity equation, we get

$$
\omega \frac{\delta n_{e}}{n_{0}}=\left(\omega_{* e}-\omega_{D e}\right) \frac{e \phi}{T_{e}}+\omega_{D e} \frac{\delta P_{e}}{P_{e}}-\frac{1}{n_{0} e} k_{\|} J_{\| e},
$$

where the terms on the right hand side represent from left to right, the divergence of the $\mathbf{E} \times \mathbf{B}$ current comprising the $\mathbf{E} \times \mathbf{B}$ convection of the background density and the compressibility of the $\mathbf{E} \times \mathbf{B}$ drift, the divergence of the diamagnetic current, and the divergence of the parallel electron current. We note that the curved inhomogeneous background magnetic field in a fluid model gives rise to magnetic drift terms $\mathbf{v}_{D}=2 T \frac{e_{\|} \times \nabla B_{0}}{q B_{0}^{2}}$ and corresponding drift frequencies $\omega_{D}=\mathbf{k}_{\perp} \cdot \mathbf{v}_{D}$, although $\mathbf{v}_{D}$ is not a fluid drift. With $\frac{\delta P_{e}}{P_{e}}$ $=\frac{\delta n_{e}}{n_{0}}+\frac{\delta T_{e}}{T_{e}}$ and substituting the expressions for $\frac{\delta n_{e}}{n_{0}}$ and $\frac{\delta T_{e}}{T_{e}}$, we can express the electron current in terms of the potentials $\phi$ and $A_{\|}$as

$$
\begin{aligned}
k_{\|} J_{\| e}= & n_{0} e\left\{\left(\omega_{* e}-\omega\right) \phi+\left[\left(\omega-\omega_{* e}\right)\left(\omega-\omega_{D e}\right)\right.\right. \\
& \left.\left.+\eta_{e} \omega_{* e} \omega_{D e}\right] \frac{A_{\|}}{k_{\|}}\right\} \frac{e}{T_{e}} .
\end{aligned}
$$




\section{B. Ion model}

The equation of motion for the ions along the magnetic field is

$$
\begin{aligned}
m_{i}\left(\frac{\partial}{\partial t}+\mathbf{v}_{* i T} \cdot \nabla\right) v_{\| i}= & q_{i}\left(-\nabla_{\|} \phi-\frac{\partial A_{\|}}{\partial t}\right)+q_{i}\left(\mathbf{v}_{* i} \times \mathbf{B}\right)_{\|} \\
& -\frac{\nabla_{\|} p_{i}}{n_{0}}-\frac{\left(\nabla \cdot \bar{\pi}_{i}\right)_{\|}}{n_{0}},
\end{aligned}
$$

where $v_{* i T}=v_{* i}\left(1+\eta_{i}\right)$ and $\bar{\pi}_{i}$ is the stress tensor. Keeping there only the gyroviscous components (Braginskii Eqs. (20) and (21)), the diamagnetic convection term on the left hand side is cancelled by an identical contribution in $\left(\nabla \cdot \bar{\pi}_{i}\right)_{\|}$. We then obtain the parallel ion velocity ${ }^{1}$

$$
v_{\| i}=\frac{v_{t h i}^{2}}{\omega}\left\{\left[k_{\|} \phi-\left(\omega-\omega_{* i T}\right) A_{\|}\right] \frac{e}{T_{i}}+k_{\|} \frac{\delta P_{i}}{P_{i}}\right\},
$$

where $v_{t h i}^{2}=\frac{T_{i}}{m_{i}}$ (ion thermal velocity), $\omega_{* i T}=\omega_{* i}\left(1+\eta_{i}\right)$, and $J_{\| i}=q_{i} n_{0} v_{\| i}=e n_{0} v_{\| i}$. We note that $v_{\| i}$ goes to zero if $\frac{v_{t h i}}{\omega / k_{\|}} \ll 1$ (ion thermal velocity much smaller than the parallel phase velocity). From the ion continuity equation, we obtain

$$
\begin{aligned}
\omega \frac{\delta n_{i}}{n_{o}}= & \left(\omega_{D i}-\omega_{* i}\right) \frac{e \phi}{T_{i}}+\omega_{D i} \frac{\delta P_{i}}{P_{i}} \\
& -k^{2} \rho_{i}^{2}\left(\omega-\omega_{* i T}\right) \frac{e \phi}{T_{i}}+\frac{1}{n_{o} e} k_{\|} J_{\| i},
\end{aligned}
$$

where $\omega_{* i}=-\frac{\omega_{* e}}{\tau}$ and $\omega_{D i}=-\frac{\omega_{D e}}{\tau}$ are the ion diamagnetic and magnetic drift frequencies, respectively $\left(\tau=\frac{T_{e}}{T_{i}}\right)$. In this equation, the FLR (Finite Larmor Radius) term is due to the polarization and stress tensor drifts, while the others have the same origin as in the electron continuity equation.

We now apply the ion energy equation and close the fluid hierarchy by using the diamagnetic heat flow for the heat flow vector (for details, see Ref. 1). Thus

$$
\frac{3}{2} n_{i}\left(\frac{\partial}{\partial t}+\mathbf{v}_{i} \cdot \nabla\right) T_{i}+p_{i} \nabla \cdot \mathbf{v}_{i}=-\nabla \cdot \mathbf{q}_{i},
$$

where $\quad \mathbf{v}_{i}=\frac{\mathbf{E} \times \mathbf{B}_{0}}{B_{0}^{2}}+\mathbf{v}_{\perp i}+\mathbf{v}_{\| i} \quad$ and $\quad \nabla \cdot \mathbf{q}_{i}=-\frac{5}{2} n_{i} \mathbf{v}_{* i} \cdot \nabla T_{i}$ $+\frac{5}{2} n_{i} \mathbf{v}_{D i} \cdot \nabla T_{i}$ (Braginskii- $\mathbf{q}_{i}$ diamagnetic heat flow). If $\nabla \cdot \mathbf{v}_{i}=-\frac{1}{n_{i}}\left(\frac{\partial n_{i}}{\partial t}+\mathbf{v}_{i} \cdot \nabla n_{i}\right)$ is substituted from the ion continuity equation, we obtain after linearisation

$$
\frac{\delta T_{i}}{T_{i}}=\frac{\frac{2}{3} \omega \frac{\delta n_{i}}{n_{i}}+\omega_{* i}\left(\frac{2}{3}-\eta_{i}\right) \frac{e \phi}{T_{i}}}{\omega-\frac{5}{3} \omega_{D i}}
$$

where the $\eta_{i}$ term on the right hand side represents the $\mathbf{E} \times \mathbf{B}$ convection of the background temperature, and the $\frac{5}{3}$ term in the denominator represents compressibility of the diamagnetic heat flow due to the magnetic field curvature. The rest of the terms (with coefficients 2/3) originate from substitution of $\nabla \cdot \mathbf{v}_{i}$ from the ion continuity equation.

Substituting $\frac{\delta T_{i}}{T_{i}}$ in (8) and (9), we may express $\frac{\delta n_{i}}{n_{i}}$ in terms of the potentials $\phi_{2}$ and $A_{\|}$. For convenience, we introduce the notation $M=\frac{v_{t h i}^{2}}{\omega}$ accounting for the finite ion mass and $R=k^{2} \rho_{i}^{2}\left(\omega-\omega_{* i T}\right)$ accounting for the finite ion Larmor radius. From (9), we then get (after substitution of $J_{\| i}$ from (8))

$$
\frac{\delta n_{i}}{n_{i}}=\frac{\left[\left(\omega_{D i}+k_{\|}^{2} M\right)\left(1+\frac{\frac{2}{3} \omega_{* i}-\eta_{i} \omega_{* i}}{\omega-\frac{5}{3} \omega_{D i}}\right)-\omega_{* i}-R\right] \frac{e \phi}{T_{i}}-k_{\|} M\left(\omega-\omega_{* i T}\right) \frac{e A_{\|}}{T_{i}}}{\omega-\left(\omega_{D i}+k_{\|}^{2} M\right)\left(1+\frac{\frac{2}{3} \omega}{\omega-\frac{5}{3} \omega_{D i}}\right)} .
$$

We here have to have in mind that $k_{\|}$is a derivation operator along the magnetic field and that $\omega_{D}$ (but not $\omega_{*}$ ) as well as all perturbations have a variation in this direction. If parallel ion motion is neglected (i.e., $M=0$ ), we recover Eqs. (10)-(13) in Ref. 10 identically, the ion density response then being expressed in terms of the electrostatic potential $\phi$ only. Electromagnetic effects, accounted for by the $A_{\|}$dependence, then enter via the quasineutrality condition and via the Ampere law.

\section{Quasineutrality}

An equation in $\phi$ and $A_{\|}$can now be derived by using quasineutrality with $\frac{\delta n_{i}}{n_{i}}=\frac{\delta n_{e}}{n_{e}}=\left(\phi-\frac{\omega-\omega_{* e}}{k_{\|}} A_{\|}\right) \frac{e}{T_{i} \tau}$. Taking the $\phi$-terms to the left hand side, we then obtain

$$
\begin{aligned}
& {\left[\frac{\omega}{\tau}+\omega_{* i}+R-\left(\omega_{D i}+k_{\|}^{2} M\right)\left(\frac{1}{\tau}+1+\frac{\frac{2}{3} \frac{\omega}{\tau}+\frac{2}{3} \omega_{* i}-\eta_{i} \omega_{* i}}{\omega-\frac{5}{3} \omega_{D i}}\right)\right] \frac{e \phi}{T_{i}}} \\
& \left.\left.=\left[\omega-\omega_{D i}\left(1+\frac{\frac{2}{3} \omega}{\omega-\frac{5}{3} \omega_{D i}}\right)\right] \frac{\omega-\omega_{* e} \frac{e A_{\|}}{\tau} k_{\|} T_{i}}{\frac{2}{3} \omega}\right) \omega-\omega_{* e}+\omega-\omega_{* i T}\right] \frac{e A_{\|}}{k_{\|} T_{i},} \\
& -k_{\|}^{2} M\left[\left(1+\frac{5}{3} \omega_{D i}\right.\right.
\end{aligned}
$$

where the M-terms represent the parallel ion velocity. 


\section{Ampere's law in parallel direction}

From (8) and $\delta T_{i}$ from (11), we obtain the parallel ion current density

$$
\begin{aligned}
J_{\| i}= & e n_{0} M\left[\left(k_{\|} \phi-\left(\omega-\omega_{* i T}\right) A_{\|}\right) \frac{e}{T_{i}}+k_{\|}\left(1+\frac{\frac{2}{3} \omega}{\omega-\frac{5}{3} \omega_{D i}}\right) \frac{\delta n_{i}}{n_{0}}\right. \\
& \left.+\frac{k_{\|}}{\omega-\frac{5}{3} \omega_{D i}}\left(\frac{2}{3} \omega_{* i}-\eta_{i} \omega_{* i}\right) \frac{e \phi}{T_{i}}\right]
\end{aligned}
$$

Applying now Ampere's law for the parallel direction using (6) for $J_{\| e}$ and operating with $k_{\|}$on both sides, we have

$$
\begin{gathered}
\nabla_{\perp}^{2} A_{\|}=-\mu_{0}\left(J_{\| e}+J_{\| i}\right), \\
k_{\|} k^{2} A_{\|}=\mu_{0} n_{0} e\left\{\left(\omega_{* e}-\omega\right) \phi+\left[\left(\omega-\omega_{* e}\right)\left(\omega-\omega_{D e}\right)\right.\right. \\
\left.\left.+\eta_{e} \omega_{* e} \omega_{D e}\right] \frac{A_{\|}}{k_{\|}}\right\} \frac{e}{T_{e}}+k_{\|} \mu_{0} J_{\| i} .
\end{gathered}
$$

Substituting (14) and $\frac{\delta n_{i}}{n_{i}}=\frac{\delta n_{e}}{n_{e}}=\left(\phi-\frac{\omega-\omega_{* e}}{k_{\|}} A_{\|}\right) \frac{e}{T_{e}}$ and rearranging terms, we get

$$
\begin{gathered}
{\left[\frac{\omega_{* e}-\omega}{\tau}+k_{\|}^{2} M\left(1+\frac{1}{\tau}+\frac{\frac{2}{3} \frac{\omega}{\tau}+\frac{2}{3} \omega_{* i}-\eta_{i} \omega_{* i}}{\omega-\frac{5}{3} \omega_{D i}}\right)\right] \frac{e \phi}{T_{i}}} \\
=\frac{k_{\|} k^{2} k_{\|}}{e n_{0} \mu_{0}} \frac{A_{\|}}{k_{\|}}-\left[\left(\omega-\omega_{* e}\right)\left(\omega-\omega_{D e}\right)+\eta_{e} \omega_{* e} \omega_{D e}\right] \frac{e A_{\|}}{k_{\|} \tau T_{i}} \\
+k_{\|}^{2} M\left[\left(1+\frac{\frac{2}{3} \omega}{\omega-\frac{5}{3} \omega_{D i}}\right) \frac{\omega-\omega_{* e}}{\tau}+\omega-\omega_{* i T}\right] \frac{e A_{\|}}{k_{\|} T_{i}},
\end{gathered}
$$

where again (as in (13)) the M-terms represent the parallel ion velocity. We note that the electrostatic approximation $\left(A_{\|} \rightarrow 0\right)$ assumes a strong background B-field, which implies a small Larmor radius and a large $\mathrm{k}$. This means that the $\frac{k_{\|} k^{2} k_{\|} A_{\|}}{e n_{0} \mu_{0} k_{\|}}$term gives a nonzero contribution. However, in the electrostatic case, the eigenvalue equation is preferably derived from (13) where the Ampere law has not been utilized.

\section{E. Eigenvalue equations}

Equations (13) and (17) form together a system of two equations in $\phi$ and $A_{\|}$. After normalization with $\omega_{* i}$, we get

$$
\begin{aligned}
& \left(\Omega_{1 \Phi}+V_{\Phi}\right) \Phi=\left(\Omega_{1 \Psi}+V_{\Psi}\right) \Psi, \\
& \left(\Omega_{2 \Phi}-V_{\Phi}\right) \Phi=\left(\Omega_{2 \Psi}-V_{\Psi}\right) \Psi,
\end{aligned}
$$

where $\Phi=\frac{e \phi}{T_{i}}, \Psi=\frac{\omega_{i} e A_{\|}}{k_{\|} T_{i}}$, and $V_{\Phi} \Phi-V_{\Psi} \Psi$ represent the (normalized) parallel ion velocity. With $\Omega=\frac{\omega}{\omega_{* i}}$, we have

$$
\begin{aligned}
& \Omega_{1 \Phi}=\frac{\Omega}{\tau}+1+k^{2} \rho_{i}^{2}\left(\Omega-1-\eta_{i}\right) \\
& -\frac{\omega_{D i}}{\omega_{* i}}\left(1+\frac{1}{\tau}+\frac{\frac{2}{3} \frac{2}{\tau}+\frac{2}{3}-\eta_{i}}{\Omega-\frac{5}{3} \frac{\omega_{D i}}{\omega_{* i}}}\right), \\
& \Omega_{1 \Psi}=\left[\Omega-\frac{\omega_{D i}}{\omega_{* i}}\left(1+\frac{\frac{2}{3} \Omega}{\Omega-\frac{5}{3} \frac{\omega_{D i}}{\omega_{* i}}}\right)\right] \frac{\Omega+\tau}{\tau}, \\
& V_{\Phi}=-\frac{k_{\|}^{2} v_{t h i}^{2}}{\omega_{* i}^{2}} \frac{1}{\Omega}\left(1+\frac{1}{\tau}+\frac{\frac{2}{3} \frac{\Omega}{\tau}+\frac{2}{3}-\eta_{i}}{\Omega-\frac{5}{3} \frac{\omega_{D i}}{\omega_{* i}}}\right), \\
& V_{\Psi}=-\frac{k_{\|}^{2} v_{t h i}^{2}}{\omega_{* i}^{2}} \frac{1}{\Omega}\left[\left(1+\frac{\frac{2}{3} \Omega}{\Omega-\frac{5}{3} \frac{\omega_{D i}}{\omega_{* i}}}\right) \frac{\Omega+\tau}{\tau}+\Omega-1-\eta_{i}\right], \\
& \Omega_{2 \Phi}=-\frac{\Omega+\tau}{\tau}, \\
& \Omega_{2 \Psi}=\frac{k_{\|} k^{2} \rho_{i}^{2} v_{A}^{2} k_{\|}}{\omega_{* i}^{2}}-\frac{\Omega+\tau}{\tau}\left(\Omega-\frac{\omega_{D e}}{\omega_{* i}}\right)+\eta_{e} \frac{\omega_{D e}}{\omega_{* i}},
\end{aligned}
$$

where $v_{A}^{2}=\frac{B_{0}^{2}}{m_{i} n_{0} \mu_{0}}$ (Alfvén velocity). We note that $\omega_{D}$ and $k^{2}=k_{\perp}^{2}$ here have a variation along the magnetic field, while the other quantities (except the potentials) do not.

\section{F. Special cases}

\section{Electrostatic approximation}

From (18) with $A_{\|}=0$, we have $\left(\Omega_{1 \Phi}+V_{\Phi}\right) \Phi=0$, i.e.,

$$
\begin{aligned}
& {\left[\frac{\Omega}{\tau}+1+k^{2} \rho_{i}^{2}\left(\Omega-1-\eta_{i}\right)\right.} \\
& \left.-\left(\frac{\omega_{D i}}{\omega_{* i}}-\frac{v_{t h i}^{2}}{\omega_{* i}^{2}} \frac{\left(\hat{e}_{\|} \cdot \nabla\right)^{2}}{\Omega}\right)\left(1+\frac{1}{\tau}+\frac{\frac{2}{3} \frac{\Omega}{\tau}+\frac{2}{3}-\eta_{i}}{\Omega-\frac{5}{3} \frac{\omega_{D i}}{\omega_{* i}}}\right)\right] \Phi=0,
\end{aligned}
$$

where we have used $j k_{\|} \sim \hat{e}_{\|} \cdot \nabla$. In the electrostatic limit, parallel ion motion is caused by $\nabla_{\|} \phi$ and $\nabla_{\|} p$ which gives a second order differential equation along the parallel direction. Here, it is not necessary to use Amperes law, since $\delta B_{\perp} \approx 0\left(A_{\|} \approx 0\right)$ (no induced E-field along $\left.B_{0}\right)$.

\section{2. $v_{\| i}=0$ approximation}

In this limit, we have $V_{\Phi}=V_{\Psi}=0$, the only $k_{\|}$-dependence now being in $\frac{k_{\|} k^{2} \rho_{i}^{2} v_{A}^{2} k_{\|}}{\omega_{* i}^{2}}$. From (18) and (19), we then get $\Phi=\frac{\Omega_{1} \Psi}{\Omega_{1 \Phi}} \Psi$ and $\left(\Omega_{2} \Psi-\Omega_{2 \Phi} \frac{\Omega_{1 \Psi}}{\Omega_{1 \Phi}}\right) \Psi=0$, i.e., 
$\left[\frac{k_{\|} k^{2} \rho_{i}^{2} v_{A}^{2} k_{\|}}{\omega_{* i}^{2}}-\frac{\Omega+\tau}{\tau}\left(\Omega-\frac{\omega_{D e}}{\omega_{* i}}\right)+\eta_{e} \frac{\omega_{D e}}{\omega_{* i}}+\frac{\Omega+\tau}{\tau} \frac{\Omega_{1 \Psi}}{\Omega_{1 \Phi}}\right] \Psi=0$.

This equation compares with, i.e., Eq. (18) in Ref. 10.

\section{Electrostatic and $v_{\| i}=0$ approximation}

From (18) with $A_{\|}=0$ and $V_{\Phi}=V_{\Psi}=0$, we get $\Omega_{1 \Phi} \Phi=0$. We then have the following second order algebraic equation in $\Omega$ :

$\frac{\Omega}{\tau}+1+k^{2} \rho_{i}^{2}\left(\Omega-1-\eta_{i}\right)-\frac{\omega_{D i}}{\omega_{* i}}\left(1+\frac{1}{\tau}+\frac{\frac{2}{3} \frac{2}{\tau}+\frac{2}{3}-\eta_{i}}{\Omega-\frac{5}{3} \frac{\omega_{D i}}{\omega_{* i}}}\right)=0$.

\section{G. Gyro-Landau fluid model}

The gyro-Landau fluid model of Refs. 26-28 includes dissipative pole approximations that account for both the ion drift resonance and the parallel ion dynamics. This model, including anisotropic pressure perturbations, has reproduced various gyrokinetic results for the $\eta_{i}$ mode with good accuracy, and it has also found wide use in nonlinear numerical simulations of plasma turbulence. In this paper, the gyroLandau fluid model corresponds to truncating the energy equation (10) according to

$$
\nabla \cdot \mathbf{q}_{i}=\nabla \cdot \mathbf{q}_{* i}+j \nu n_{i} \frac{3}{2} \delta T_{i},
$$

where

$$
j \nu=\left(j \sigma_{t} \frac{3 \sqrt{2}}{4}+\frac{3}{4}\right) \omega_{D i}+j \sigma_{s} \frac{2}{\sqrt{\pi}} k_{\|} v_{t h i},
$$

and

$$
\begin{gathered}
\sigma_{t}=\frac{\omega_{D i}}{\left|\omega_{D i}\right|}, \\
\sigma_{s}=\frac{k_{\|}}{\left|k_{\|}\right|} .
\end{gathered}
$$

Here, the $\omega_{D i}$ term simulates the magnetic drift resonance, and the $k_{\|}$term simulates the ion Landau resonance. Adding the dissipative term $j \nu$ in the energy equation (10) gives the temperature response

$$
\frac{\delta T_{i}}{T_{i}}=\frac{\frac{2}{3} \omega \frac{\delta n_{i}}{n_{i}}+\omega_{* i}\left(\frac{2}{3}-\eta_{i}\right) \frac{e \phi}{T_{i}}}{j \nu+\omega-\frac{5}{3} \omega_{D i}}
$$

and in Eqs. (12)-(26), the denominators $\omega-\frac{5}{3} \omega_{D i}$ and $\Omega-\frac{5}{3} \frac{\omega_{D i}}{\omega_{* i}}$ will be modified according to

$$
\begin{gathered}
\omega-\frac{5}{3} \omega_{D i} \rightarrow j \nu+\omega-\frac{5}{3} \omega_{D i}, \\
\Omega-\frac{5}{3} \frac{\omega_{D i}}{\omega_{* i}} \rightarrow \frac{j \nu}{\omega_{* i}}+\Omega-\frac{5}{3} \frac{\omega_{D i}}{\omega_{* i}} .
\end{gathered}
$$

In Ref. 29, our two-fluid model, excluding parallel ion velocity effects, has been compared with a gyrofluid model, i.e., adding ion drift resonance effects $\left(\omega_{D i}\right.$ term in $\left.j \nu\right)$, and a gyrokinetic model. It is shown that the two-fluid model is able to reproduce the instabilities qualitatively, while lack of quantitative agreement stems from the FLR expansion and neglect of dissipative ion drift resonance effects. These are important since the extended eigenmodes experience large values of the kinetic parameters $k^{2}(\theta) \rho_{i}^{2}$ and $\frac{\omega_{D}(\theta)}{\omega_{*}}$ for large $\theta$. The gyrofluid model is shown to be able to reproduce the eigenvalues quantitatively for small values of the FLR parameter. The discrepancy for large values of this parameter may be due to the use of isotropic pressure perturbations. The more complete two-pressure version of the gyrofluid model (Refs. 26-28) may be adequate in this region.

In the present work, we focus on the effects of parallel ion velocity and neglect the ion drift resonance but keep the ion Landau resonance.

\section{H. Ballooning mode equations}

Assuming an axisymmetric toroidal (Tokamak) geometry with a low beta equilibrium with circular flux surfaces and a high mode number approximation, we may use the ballooning mode formalism where the spatial perturbation is assumed to be of the form ${ }^{10,34}$

$$
\Phi=\Phi_{n}(r, \theta) e^{\left[i n \int^{\theta} \nu\left(r, \theta^{\prime}\right) d \theta^{\prime}-i n \zeta\right]} .
$$

Here, the exponential is the fast varying part, $r, \theta$, and $\zeta$ are the (orthogonal) toroidal coordinates, and $\mathrm{n}$ is the toroidal mode number. The variations of $\omega_{D}, k=k_{\perp}$, and $k_{\|}$with the poloidal angle $\theta$ along the twisted magnetic field are expressed by the following relations:

$$
\begin{gathered}
\omega_{D} \rightarrow \epsilon_{n} \omega_{*}[\cos \theta+(s \theta-\alpha \sin \theta) \sin \theta]=\epsilon_{n} \omega_{*} g(\theta), \\
k_{\perp}^{2} \rightarrow k_{\theta}^{2}\left[1+(s \theta-\alpha \sin \theta)^{2}\right]=k_{\theta}^{2} \tilde{r}(\theta), \\
k_{\|} \rightarrow-j \frac{1}{q R} \frac{\partial}{\partial \theta}
\end{gathered}
$$

Here, $\epsilon_{n}=\frac{\omega_{D}}{\omega_{*}}=\frac{2 L_{n}}{R}, \alpha=\frac{\beta q^{2}}{\epsilon_{n}}\left(1+\eta_{e}+\frac{1+\eta_{i}}{\tau}\right), \beta=\beta_{i}(1+\tau)$, $\beta_{i}=\frac{2 \mu_{0} n_{0} T_{i}}{B^{2}}$ is the ion plasma beta, $q$ is the safety factor, and $s=\frac{r q^{\prime}}{q}$ is the magnetic shear. By means of these relations, the two coupled equations (18) and (19) may be transformed into two equations in the poloidal angle $\theta$. Doing so, we obtain after some algebra (see the Appendix for details)

$$
\begin{gathered}
\Psi^{\prime \prime}=f_{1}\left(\theta, \Psi, \Psi^{\prime}, \Phi, \Omega\right), \\
\Phi^{\prime \prime}=f_{2}\left(\theta, \Psi, \Psi^{\prime}, \Psi^{\prime \prime}, \Phi, \Phi^{\prime}, \Omega\right),
\end{gathered}
$$

where prime and bis mean derivatives with respect to $\theta$.

\section{Semilocal approximation}

An approximate solution of the eigenvalue equations can be obtained by using a semilocal theory where the eigenfunctions are approximated with ${ }^{37-40}$ 


$$
\Phi(\theta), \Psi(\theta) \propto \frac{1+\cos (\theta)}{\sqrt{3 \pi}}, \quad|\theta| \leq \pi .
$$

This eigenfunction has earlier been used for ballooning modes in a two-fluid description ${ }^{10,25}$ with good qualitative agreement. By making the substitutions $k_{\|} \rightarrow-j \frac{1}{q R}\left\langle\frac{\partial}{\partial \theta}\right\rangle$, $k_{\perp}^{2} \rightarrow\left\langle k_{\perp}^{2}\right\rangle$ and $\omega_{D} \rightarrow\left\langle\omega_{D}\right\rangle$, where \langle\rangle denotes averages over the approximate eigenfunction, we may, from (18)-(25), obtain a polynomial equation in $\omega$ where the norms are

$$
\begin{gathered}
\left\langle k_{\perp}^{2}\right\rangle=\int_{-\pi}^{\pi} \Phi k_{\perp}^{2} \Phi d \theta \\
=k_{\theta}^{2}\left[1+\left(\frac{\pi^{2}}{3}-2.5\right) s^{2}-\frac{10}{9} \alpha s+\frac{5}{12} \alpha^{2}\right] \\
\left\langle k_{\|}^{2}\right\rangle=\int_{-\pi}^{\pi} \Phi k_{\|}^{2} \Phi d \theta=\frac{1}{3(q R)^{2}} \\
\left\langle\omega_{D}\right\rangle=\int_{-\pi}^{\pi} \Phi \omega_{D} \Phi d \theta=\epsilon_{n} \omega_{*}\left(\frac{2}{3}+\frac{5}{9} s-\frac{5}{12} \alpha\right) \\
\left\langle k_{\|} k_{\perp}^{2} k_{\|}\right\rangle=\frac{k_{\theta}^{2}}{3(q R)^{2}}\left[1+s^{2}\left(\frac{\pi^{2}}{3}-0.5\right)-\frac{8}{3} \alpha s+\frac{3}{4} \alpha^{2}\right]
\end{gathered}
$$

In this work, however, we choose to use the semilocal approximation only in the evaluation of the ion Landau resonance contribution in order to avoid a system of equations (40) and (41) with higher than second order derivatives. In Sec. III, we give results of the numerical solution of this eigenvalue system using the ballooning mode formalism.

\section{NUMERICAL RESULTS}

The system of two coupled differential equations in the potentials $\Phi$ and $\Psi$ (Eqs. (18)-(25)) has been solved numerically. By means of the ballooning mode formalism, the coupled differential equations (A2) and (A3) in the extended poloidal angle $\theta$ are derived (see the Appendix). To solve this system, a shooting code has been developed where the two potentials are shot simultaneously. The code uses Runge Kutta and Muller methods, and the usual ballooning mode boundary conditions $\Phi^{\prime}(0)=\Psi^{\prime}(0)=0$ and $\Phi, \Psi \rightarrow 0$ as $\theta \rightarrow \infty$ have been applied. ${ }^{34}$ The following parameters have been chosen. $\tau=1, \eta_{i}=\eta_{e}, k^{2} \rho^{2}=0.1$ (mode number with largest growth rate in several studies ${ }^{10}$ shown to fulfil $k \rho \approx 0.3$ ), $\mathrm{q}=2$ (safety factor used in, e.g., Refs. 10 and 11 ), and $\mathrm{s}=1$. As a comparison, some of the parameters used in the study of the Cyclone DIII- $\mathrm{D}^{36}$ high confinement shot 81499 were $\tau=1, \eta_{i}=3.1, \mathrm{q}=1.4$, and $\mathrm{s}=0.8$. We show the results both with (glf) and without the ion Landau resonance taken into account as well as the case without parallel ion velocity.

\section{A. Effects of finite beta}

Fig. 1 displays the normalized frequency $\frac{\omega}{\omega_{x e}}$ (growth rate and real frequency) as a function of $\beta$ for the cases with and without parallel ion motion. We note the sudden $\beta$ stabilization of the pure ITG mode which is characteristic and has

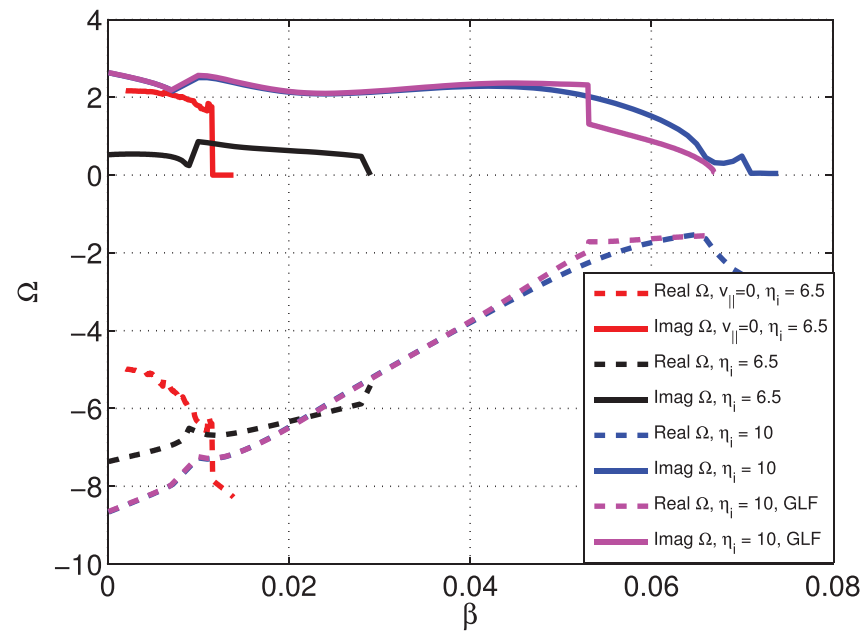

FIG. 1. The normalized mode frequency $\Omega=\frac{\omega}{\omega_{* e}}$ (growth rate-positive and real frequency-negative) as a function of $\beta$ for the cases with and without parallel ion motion. GLF $=$ ion Landau resonance taken into account. $\epsilon_{n}=2.5, \eta_{i}=\eta_{e}=6.5,10, \tau=1, k^{2} \rho^{2}=0.1, q=2, s=1$.

been verified in previous studies ${ }^{12,13}$ and is related to the strong ballooning and electrostatic character of the mode. With parallel ion motion, marginal stability is shifted upwards, and the growth rate is reduced. The real frequency is of the same order as the diamagnetic frequency and the ion transit frequency $\omega_{t i}=k_{\|} v_{\| i}=\frac{1}{q R} v_{\| i}$. (With the parameters in Figure 1, we get $\frac{\omega}{\omega_{i i}}=0.5 \frac{\omega}{\omega_{* e}}$.) We note that inclusion of the ion Landau resonance (glf) does not have much influence on neither the frequency nor the growth rate except closer to marginal stability where a mode switch creates a step in the scaling.

Fig. 2(a) displays the electrostatic and electromagnetic potentials $|\Phi|$ and $|\Psi|$ as a function of the extended poloidal angle $\theta$ for the case without parallel ion motion. ( $\Phi$ normalized to one at $\theta=0$.) We note the strong localization of $\Phi$ around $\theta=0$ (strong ballooning). This localization is released by the influence of the ion acoustic mode when parallel ion motion is present (Fig. 2(b)). The $\theta$ dependence for the case when the ion Landau resonance effect is added is similar to Fig. 2(b) and so not displayed here. We note that $\Phi$ shows a magnetic drift resonance (where $\theta$ fulfils $\left.\operatorname{Re}(\omega)-\frac{5}{3} \omega_{D}=0\right)$. This resonance does not show in the electromagnetic potential. We also note that $\frac{\Psi}{\Phi}$ is about two orders of magnitude smaller than $\beta$ without parallel ion motion but the order of $\beta$ with parallel ion motion.

\section{B. Effects of ITG}

Fig. 3 displays the normalized frequency $\frac{\omega}{\omega_{x e}}$ (growth rate and real frequency) as a function of $\eta_{i}$ for the cases with and without parallel ion motion. We note that the pure ITG mode, being driven by temperature gradients, is destabilized by $\eta_{i}$. With parallel ion motion, the destabilization is upshifted in $\eta_{i}$, and both the growth rate and the frequency are smaller than without the parallel motion. This is also seen in Ref. 11, although finite $\beta$ effects are absent there. With the ion Landau resonance effect (glf), a couple of mode shifts occur (steps in the curve), and the stabilization is upshifted in $\eta_{i}$ as compared to the case without glf. The 

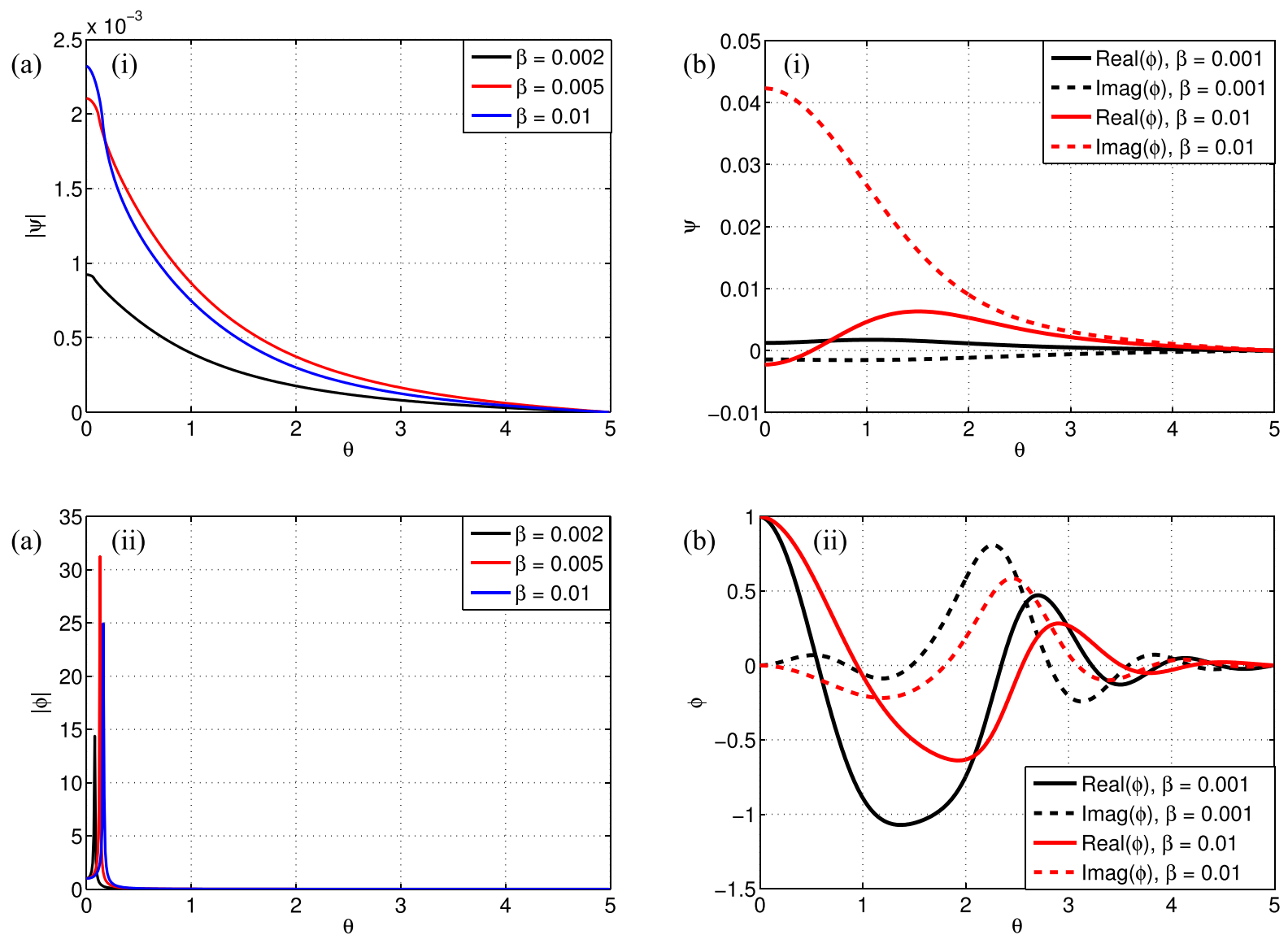

FIG. 2. (a) The electromagnetic (i) and electrostatic (ii) potentials $|\Psi|$ and $|\Phi|$ as a function of the extended poloidal angle $\theta$ for the case without parallel ion motion. $\epsilon_{n}=2.5, \eta_{i}=\eta_{e}=6.5, \tau=1, k^{2} \rho^{2}=0.1, q=2, s=1, \beta=0.002,0.005,0.01$. (b) The electromagnetic (i) and electrostatic (ii) potentials $\Psi$ and $\Phi$ as a function of the extended poloidal angle $\theta$ for the case with parallel ion motion. Parameters the same as in (a) except $\eta_{i}=\eta_{e}=10$.

frequencies on the other hand are almost exactly the same for the two cases.

The dependence of the potentials on the extended poloidal angle $\theta$ shows a similar behaviour as in Figs. 2(a) and 2(b). Fig. 4(a) displays the electrostatic and electromagnetic potentials $\Phi$ and $\frac{\Psi}{\beta}$ for the case with parallel ion motion and two values of $\eta_{i}$ ( $\Phi$ normalized to one at $\theta=0$ ). Fig. 4(b) is the corresponding figure for the case including the glf

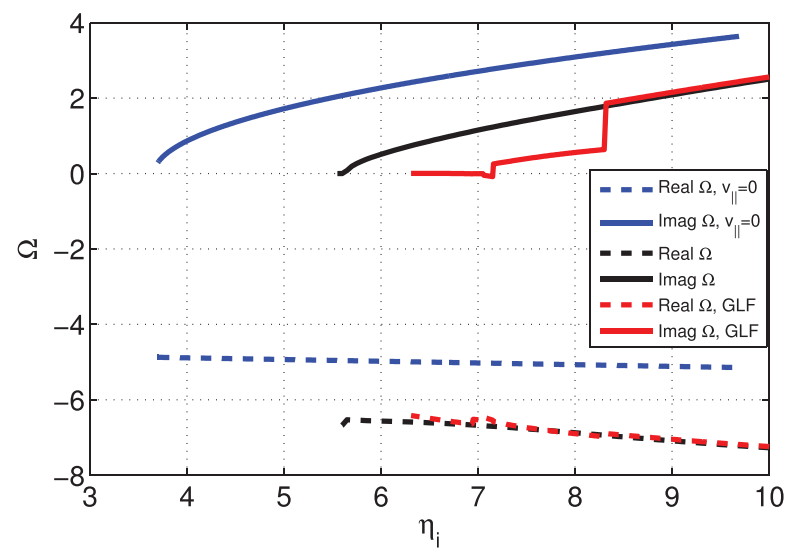

FIG. 3. The normalized mode frequency $\Omega=\frac{\omega}{\omega_{* e}}$ (growth rate-positive and real frequency-negative) as a function of $\eta_{i}=\eta_{e}$ for the cases with and without (blue curves) parallel ion motion. $\epsilon_{n}=2.5, \tau=1, k^{2} \rho^{2}=0.1$, $q=2, s=1, \beta=0.01$. contribution. We note the magnetic drift resonance at $\theta \approx 2.5$, where $\omega_{D} \propto \cos (\theta)+(s \theta-\alpha \sin (\theta)) \sin (\theta)$ passes through zero and there is very sensitive to small variations in 0. The resonance is weaker in Fig. 4(a) than in Fig. 4(b), and there also more pronounced for the lower $\eta_{i}$ value (closer to marginal stability). We also note the difference in mode character between $\eta_{i}=8$ and $\eta_{i}=10$ in Fig. 4(b)—a mode shift shown in Fig. 3 occurring at $\eta_{i} \approx 8.3$. We also note that $\frac{\Psi}{\Phi}$ is of the order of $\beta$ in both of the figures (i.e., with and without the glf contribution).

\section{Effects of toroidicity- $\epsilon_{n}$}

Fig. 5 displays the normalized frequency $\frac{\omega}{\omega_{* e}}$ as a function of $\epsilon_{n}=\frac{2 L_{n}}{R}$ for the cases with and without parallel ion motion. With $v_{\|}=0$, the mode is extended towards both low and high values of $\epsilon_{n}{ }^{10,25}$ With parallel ion motion, however, the growth rate is decreased and the mode ultimately stabilized with $\epsilon_{n}$. This is due to the increase of $k_{\|} \propto \frac{1}{q R}$ and as a consequence enhanced effect of the parallel motion. The toroidicity $\left(\propto \frac{1}{R}\right)$ then may be interpreted as the driving source of the acoustic mode with an $\epsilon_{n}$ stabilization rate stronger than for the pure $\eta_{i}$ mode. We note the almost identical growth rates for the cases with and without glf contribution and the slightly larger frequency for the former case. In Fig. 5, we should also note the $\epsilon_{n}$ dependence of the 

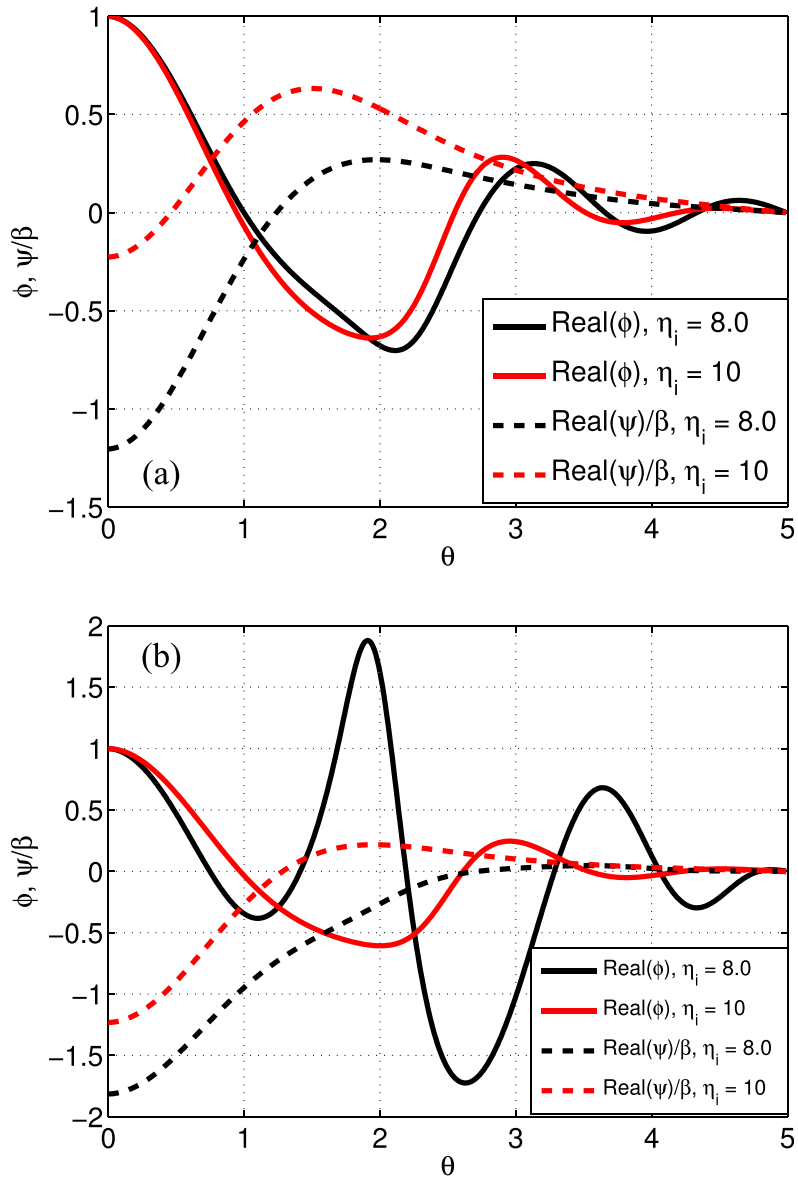

FIG. 4. (a) The electrostatic and electromagnetic potentials $\Phi$ and $\frac{\Psi}{\beta}$ as a function of the extended poloidal angle $\theta$ for the case with parallel ion motion. $\epsilon_{n}=2.5, \tau=1, k^{2} \rho^{2}=0.1, q=2, s=1, \beta=0.01, \eta_{i}=\eta_{e}=8,10$. (b) The electrostatic and electromagnetic potentials $\Phi$ and $\frac{\Psi}{\beta}$ as a function of the extended poloidal angle $\theta$ for the case with parallel ion motion and glf contribution. Parameters the same as in (a).

diamagnetic normalization frequency $\omega_{* e}\left(\omega_{* e} \propto \frac{1}{\epsilon_{n}}\right)$ which adds to the $\Omega$ dependence of $\epsilon_{n}$.

Fig. 6(a) shows the potentials $\Phi$ (expanded scale) and $\Psi$ as a function of $\theta$ for the case without parallel ion motion for a couple of $\epsilon_{n}$ values. We note, as in Fig. 2(a),

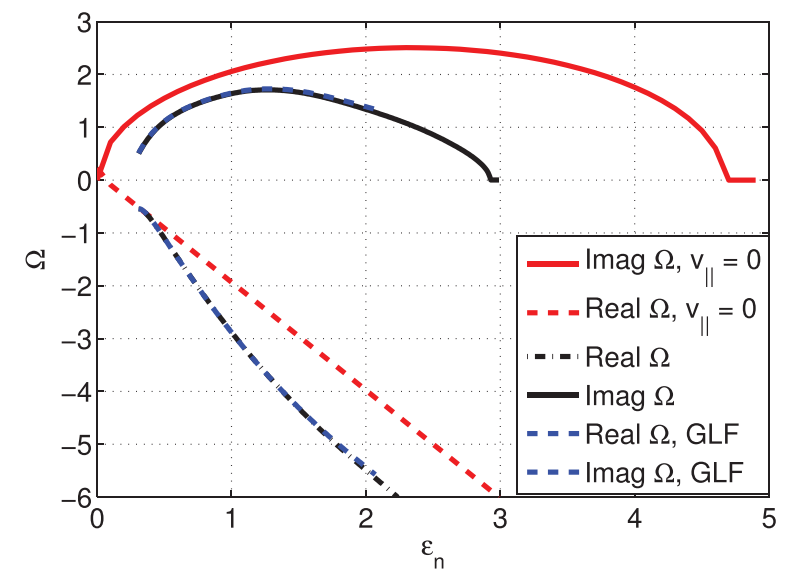

FIG. 5. The normalized mode frequency $\Omega=\frac{\omega}{\omega_{e x}}$ (growth rate-positive and real frequency-negative) as a function of $\epsilon_{n}$ for the cases with and without parallel ion motion. $\eta_{i}=\eta_{e}=6.5, \tau=1, k^{2} \rho^{2}=0.1, q=2, s=1, \beta=0.01$. the strong localization of the pure $\eta_{i}$ mode to the outer side of the torus (strong ballooning-electrostatic potential $\Phi$ large at $\theta$ close to zero) and also mainly electrostatic character $\left(\frac{\Psi}{\Phi}\right.$ small $)$.

Fig. 6(b) shows the potentials for the case with parallel ion motion and ion Landau resonance effect included. Similar to Figs. 2(b), 4(a), and 4(b), the magnetic resonance in $\Phi$ at $\operatorname{Re}(\omega)-\frac{5}{3} \omega_{D}=0$ is also present here. The corresponding modes for the case without glf show a $\theta$ dependence very similar to Fig. 6(b) and are excluded here.

\section{SUMMARY}

An advanced fluid model has been extended to include effects of parallel ion dynamics in toroidal (Tokamak) geometry. The analytical result is a system of two coupled second order differential equations in the electrostatic and electromagnetic potentials.

The model has earlier ${ }^{10,30}$ been compared with, e.g., a local kinetic model and a model where dissipative waveparticle resonances are taken into account by adding an imaginary part (gyro-Landau contributions) to the heat flow $\left(\nabla \cdot \mathbf{q}_{i}\right)$ to represent all higher order moments in the fluid hierarchy. This should increase the reliability of the model. However, since nonlinear effects in velocity space have a tendency to take particles out of resonance, we may expect nonlinear effects to decrease the imaginary part on the transport time scale in a nonlinearly saturated state.

Our mode analysis is based on the ballooning formalism with a full shooting technique (four quantities-real and imaginary parts of the electrostatic and electromagnetic potentials are shot simultaneously). Approximations in the (linearised) basic equations are avoided and no assumptions (except the semilocal) are made a priori about, e.g., strong ballooning $(\theta=0)$ or structure (Gauss, harmonic) of the mode in ballooning space.

The finite parallel ion velocity allows an acoustic mode to be set up and interact with the ion temperature gradient mode. It is shown that this removes and shifts the characteristic ITG mode sharp $\beta$ stabilization upwards and also reduces both the frequency and the growth rate. The localization to the outer part of the torus (strong ballooning), characteristic for the pure ITG interchange mode, and the sharp resonance in the electrostatic potential in the ballooning space are relaxed and shifted outwards by the parallel ion motion, i.e., the ballooning is weakened by the nonlocal contribution $\left(\propto k_{\|}\right)$caused by the parallel ion motion. The frequency of the mode may be of the order of the ion transit frequency. The growth rate, however, is reduced in relation to the pure $\left(v_{\|}=0\right)$ ITG mode. The ion Landau resonance is taken into account in our model by adapting the Landau part of a gyro-Landau fluid model. ${ }^{11,26-28}$ It is shown that this complement (glf) may enhance the $\eta_{i}$ stabilization but otherwise that it gives growth, frequency, and ballooning properties which are similar to the case without ion Landau resonance being taken into account. 
(a)
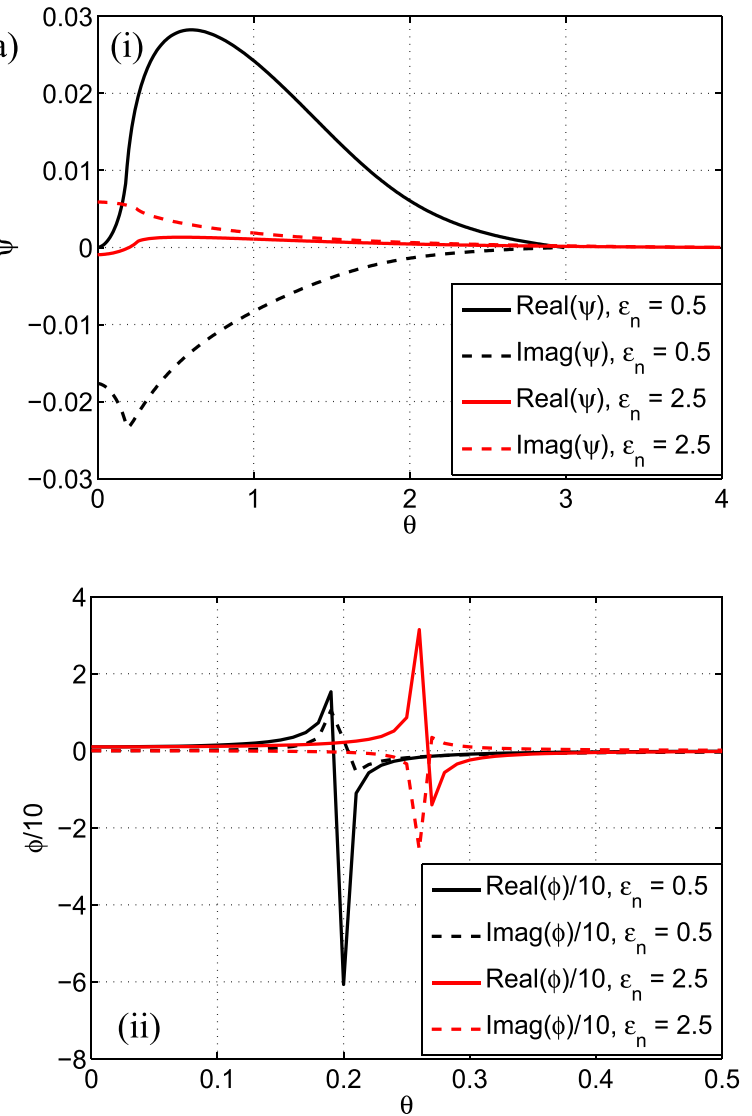

(b)
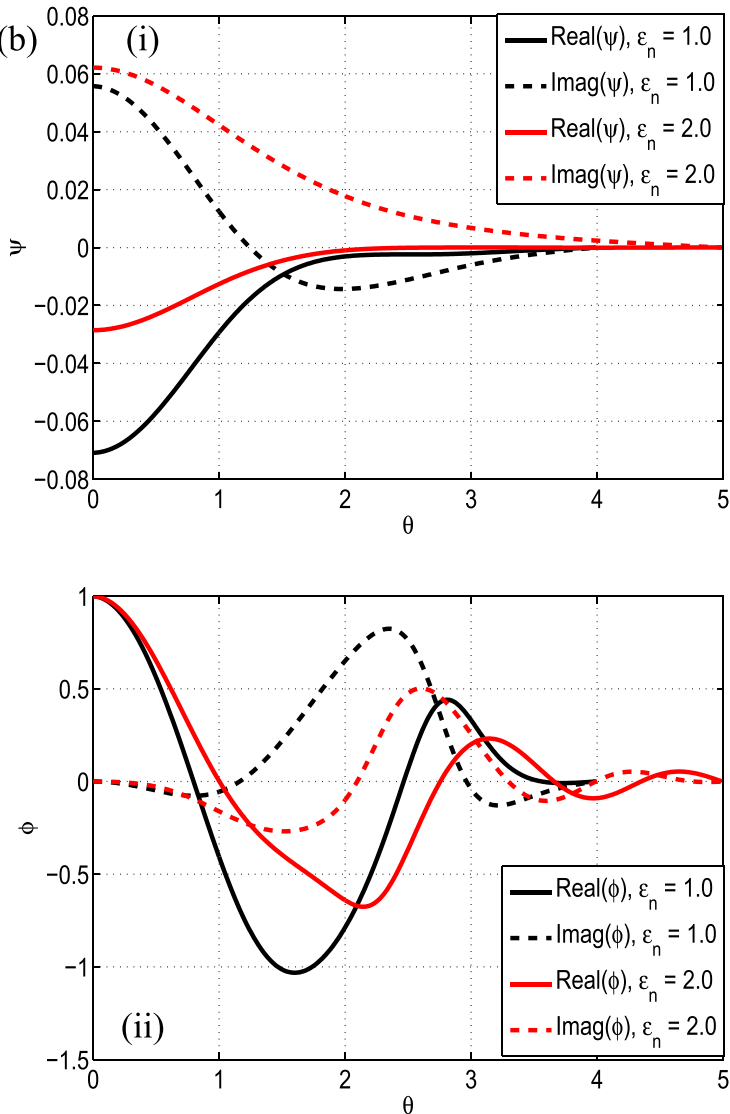

FIG. 6. (a) The electromagnetic (i) and electrostatic (ii) potentials $P$ si and $\frac{\Phi}{10}$ as a function of the extended poloidal angle $\theta$ for the case without parallel ion motion. $\eta_{i}=\eta_{e}=6.5, \tau=1, k^{2} \rho^{2}=0.1, q=2, s=1, \beta=0.01, \epsilon_{n}=0.5,2.5$. (b) The electromagnetic (i) and electrostatic (ii) potentials $\Psi$ and $\Phi$ as a function of the extended poloidal angle $\theta$ for the case with parallel ion motion and glf contribution. Parameters the same as in (a) and $\epsilon_{n}=1,2$.

The stabilization of the pure ITG mode with decreasing $\eta_{i}\left(\eta_{i}=\frac{L_{n}}{L_{T}}\right)$ is shown to be feasible also in the presence of ion transit effects with an upshifted $\eta_{i}$ stabilization, which is more pronounced when including the ion Landau resonance (glf). With our choice of parameters (similar to those used in, e.g., the Cyclone DIII-D study ${ }^{36}$ ), the mode including parallel motion has a reduced frequency and growth rate compared to the case without parallel motion. This trend is also seen in Ref. 11, although finite $\beta$ effects are absent there. Similarly, we find the $\epsilon_{n}$ dependence $\left(\epsilon_{n}=\frac{2 L_{n}}{R}\right)$ to be different compared to the pure ITG mode. The stabilization with $\epsilon_{n}$ is faster, and the frequency and growth rate are reduced. Addition of the ion Landau resonance (glf) does not change this result.

The parallel ion motion enhances electromagnetic effects, the relation between the electromagnetic and electrostatic potentials being about one or two orders of magnitude larger than without the parallel motion. In general, the simultaneous shooting of the electrostatic and electromagnetic potentials towards large values of the extended poloidal angle in the ballooning formalism is not an easy task. This is particularly true for the electrostatic potential which, contrary to the electromagnetic potential, strongly exhibits the magnetic drift resonance.

Altogether, the results are interpreted as a tendency for the acoustic mode to set its mark on the dependencies studied in this paper. Further studies comparing results from this two-fluid model and gyrokinetic models as well as related experiments are planned to be the scope of future work.

\section{ACKNOWLEDGMENTS}

The authors gratefully acknowledge the staffs at the Department of Earth and Space Sciences and the Department of Nuclear Engineering at Chalmers University of Technology for suggestions and support.

\section{APPENDIX: EQUATIONS IN THE POLOIDAL ANGLE}

We note that the parallel ion motion terms in (18) and (19) appear as "mirrors," i.e., identically but with opposite signs. If the two equations are added, we obtain

$$
\left(\Omega_{1 \Phi}+\Omega_{2 \Phi}\right) \Phi=\left(\Omega_{1 \Psi}+\Omega_{2 \Psi}\right) \Psi,
$$

and with (20)-(25) and (30)-(32)

$$
\begin{aligned}
k_{\theta}^{2} \tilde{r} & \rho_{i}^{2}\left(\Omega-1-\eta_{i}\right) \Phi-\epsilon_{n} g\left[1+\frac{1}{\tau}+\frac{1}{N}\left(\frac{2}{3} \frac{\Omega}{\tau}+\frac{2}{3}-\eta_{i}\right)\right] \Phi \\
= & -\left(\frac{1}{q R}\right)^{2} \frac{k_{\theta}^{2} \rho_{i}^{2} v_{A}^{2}}{\omega_{* i}^{2}}\left(\tilde{r}^{\prime} \Psi^{\prime}+\tilde{r} \Psi^{\prime \prime}\right) \\
& -\epsilon_{n} g\left[\frac{\Omega+\tau}{\tau}\left(\tau+1+\frac{2}{3} \frac{\Omega}{N}\right)+\eta_{e} \tau\right] \Psi,
\end{aligned}
$$


where $N=\Omega-\frac{5}{3} \epsilon_{n} g$ and $\tilde{r}^{\prime}=\frac{\partial \tilde{r}}{\partial \theta}=2(s \theta-\alpha \sin \theta)(s-\alpha \cos \theta)$. From (18), we obtain

$$
\begin{aligned}
& \frac{\Omega+\tau}{\tau} \Phi+k_{\theta}^{2} \tilde{r} \rho_{i}^{2}\left(\Omega-1-\eta_{i}\right) \Phi \\
& \quad-\epsilon_{n} g\left[1+\frac{1}{\tau}+\frac{1}{N}\left(\frac{2}{3} \frac{\Omega}{\tau}+\frac{2}{3}-\eta_{i}\right)\right] \Phi \\
& \quad+\left(\frac{1}{q R}\right)^{2} \frac{v_{t h i}^{2}}{\omega_{* i}^{2}} \frac{1}{\Omega}\left[\left(1+\frac{1}{\tau}\right) \Phi^{\prime \prime}+\left(\frac{2}{3} \frac{\Omega}{\tau}+\frac{2}{3}-\eta_{i}\right)\left(\frac{\Phi}{N}\right)^{\prime \prime}\right] \\
& =\left[\Omega-\epsilon_{n} g\left(1+\frac{2}{3} \frac{\Omega}{N}\right)\right] \frac{\Omega+\tau}{\tau} \Psi \\
& \quad+\left(\frac{1}{q R}\right)^{2} \frac{v_{t h i}^{2}}{\omega_{* i}^{2}} \frac{1}{\Omega}\left[\left(\frac{\Omega}{\tau}+\Omega-\eta_{i}\right) \Psi^{\prime \prime}+\frac{\Omega+\tau}{\tau} \frac{2}{3} \Omega\left(\frac{\Psi}{N}\right)^{\prime \prime}\right] .
\end{aligned}
$$

From (19), we obtain

$$
\begin{aligned}
- & \frac{\Omega+\tau}{\tau} \Phi \\
& -\left(\frac{1}{q R}\right)^{2} \frac{v_{t h i}^{2}}{\omega_{* i}^{2}} \frac{1}{\Omega}\left[\left(1+\frac{1}{\tau}\right) \Phi^{\prime \prime}+\left(\frac{2}{3} \frac{\Omega}{\tau}+\frac{2}{3}-\eta_{i}\right)\left(\frac{\Phi}{N}\right)^{\prime \prime}\right] \\
= & \left(\frac{1}{q R}\right)^{2} \frac{k_{\theta}^{2} \rho_{i}^{2} v_{A}^{2}}{\omega_{* i}^{2}}\left(\tilde{r}^{\prime} \Psi^{\prime}+\tilde{r} \Psi^{\prime \prime}\right) \\
& -\left[\frac{\Omega+\tau}{\tau}\left(\Omega+\tau \epsilon_{n} g\right)+\eta_{e} \tau \epsilon_{n} g\right] \Psi \\
& -\left(\frac{1}{q R}\right)^{2} \frac{v_{t h i}^{2}}{\omega_{* i}^{2}} \frac{1}{\Omega}\left[\left(\frac{\Omega}{\tau}+\Omega-\eta_{i}\right) \Psi^{\prime \prime}+\frac{\Omega+\tau}{\tau} \frac{2}{3} \Omega\left(\frac{\Psi}{N}\right)^{\prime \prime}\right],
\end{aligned}
$$

where

$$
\begin{gathered}
\left(\frac{\Phi}{N}\right)^{\prime \prime}=\frac{2\left(N^{\prime}\right)^{2}}{N^{3}} \Phi-\frac{N^{\prime \prime}}{N^{2}} \Phi-2 \frac{N^{\prime}}{N^{2}} \Phi^{\prime}+\frac{\Phi^{\prime \prime}}{N}, \\
N^{\prime}=-\frac{5}{3} \epsilon_{n} g^{\prime}=-\frac{5}{3} \epsilon_{n}[(-1+s) \sin \theta+s \theta \cos \theta-\alpha \sin 2 \theta], \\
N^{\prime \prime}=-\frac{5}{3} \epsilon_{n} g^{\prime \prime} \\
=-\frac{5}{3} \epsilon_{n}[(-1+2 s) \cos \theta-s \theta \sin \theta-2 \alpha \cos 2 \theta] .
\end{gathered}
$$

The $\left(\frac{1}{q R}\right)^{2} \frac{v_{t h i}^{2}}{\omega^{2}}$ factor (parallel ion velocity contribution) may be written $\left(\epsilon_{n}=\frac{2 L_{n}}{R}\right)$

$$
\left(\frac{1}{q R}\right)^{2} \frac{v_{t h i}^{2}}{\omega_{* i}^{2}}=\left(\frac{1}{q R}\right)^{2} \frac{v_{t h i}^{2}}{\left(k_{\theta} \frac{\rho_{i}}{L_{n}} v_{t h i}\right)^{2}}=\left(\frac{\epsilon_{n}}{2 q}\right)^{2} \frac{1}{k_{\theta}^{2} \rho_{i}^{2}} .
$$

Similarly, we may write in the Alfvén terms

$$
\left(\frac{1}{q R}\right)^{2} \frac{k_{\theta}^{2} \rho_{i}^{2} v_{A}^{2}}{\omega_{* i}^{2}}=\left(\frac{\epsilon_{n}}{2 q}\right)^{2} \frac{v_{A}^{2}}{v_{t h i}^{2}}=\left(\frac{\epsilon_{n}}{2 q}\right)^{2} \frac{2}{\beta_{i}},
$$

\footnotetext{
where $\beta_{i}=\frac{2 \mu_{0} n T_{i}}{B_{0}^{2}}$ is the ion plasma beta. By introducing
}

$$
\chi=\left[1+\frac{1}{\tau}+\frac{1}{N}\left(\frac{2}{3} \frac{\Omega}{\tau}+\frac{2}{3}-\eta_{i}\right)\right] \Phi .
$$

Eqs. (A2) and (A3) may also be written

$$
\begin{aligned}
& {\left[\epsilon_{n} g-\frac{k_{\theta}^{2} \tilde{r} \rho_{i}^{2}\left(\Omega-1-\eta_{i}\right)}{1+\frac{1}{\tau}+\frac{1}{N}\left(\frac{2}{3} \frac{\Omega}{\tau}+\frac{2}{3}-\eta_{i}\right)}\right] \chi} \\
& =\left(\frac{\epsilon_{n}}{2 q}\right)^{2} \frac{2}{\beta_{i}}\left(\tilde{r}^{\prime} \Psi^{\prime}+\tilde{r} \Psi^{\prime \prime}\right) \\
& \quad+\epsilon_{n} g\left[\frac{\Omega+\tau}{\tau}\left(\tau+1+\frac{2}{3} \frac{\Omega}{N}\right)+\eta_{e} \tau\right] \Psi,
\end{aligned}
$$

and

$$
\begin{aligned}
& -\left[\epsilon_{n} g-\frac{\frac{\Omega+\tau}{\tau}+k_{\theta}^{2} \tilde{r} \rho_{i}^{2}\left(\Omega-1-\eta_{i}\right)}{1+\frac{1}{\tau}+\frac{1}{N}\left(\frac{2}{3} \frac{\Omega}{\tau}+\frac{2}{3}-\eta_{i}\right)}\right] \chi+\left(\frac{\epsilon_{n}}{2 q}\right)^{2} \frac{1}{k_{\theta}^{2} \rho_{i}^{2}} \frac{1}{\Omega} \chi^{\prime \prime} \\
& =\left[\Omega-\epsilon_{n} g\left(1+\frac{2}{3} \frac{\Omega}{N}\right)\right] \frac{\Omega+\tau}{\tau} \Psi \\
& +\left(\frac{\epsilon_{n}}{2 q}\right)^{2} \frac{1}{k_{\theta}^{2} \rho_{i}^{2}} \frac{1}{\Omega}\left[\left(\frac{\Omega}{\tau}+\Omega-\eta_{i}\right) \Psi^{\prime \prime}+\frac{\Omega+\tau}{\tau} \frac{2}{3} \Omega\left(\frac{\Psi}{N}\right)^{\prime \prime}\right]
\end{aligned}
$$

Substituting $\chi$ from (A11) into (A12), we would get a fourth order equation in $\Psi$ (instead of two coupled second order equations).

\section{Special cases}

\section{a. Electrostatic approximation}

From (26), we obtain

$\left[\frac{\Omega+\tau}{\tau}+k_{\theta}^{2} \tilde{r} \rho_{i}^{2}\left(\Omega-1-\eta_{i}\right)\right] \Phi-\epsilon_{n} g \chi+\left(\frac{1}{q R}\right)^{2} \frac{v_{t h i}^{2}}{\omega_{* i}^{2}} \frac{1}{\Omega} \chi^{\prime \prime}=0$,

where

$$
\chi=\left[1+\frac{1}{\tau}+\frac{1}{N}\left(\frac{2}{3} \frac{\Omega}{\tau}+\frac{2}{3}-\eta_{i}\right)\right] \Phi .
$$

Substituting $\Phi$, we then get

$$
\left(\frac{1}{q R}\right)^{2} \frac{v_{t h i}^{2}}{\omega_{* i}^{2}} \frac{1}{\Omega} \chi^{\prime \prime}-\left[\epsilon_{n} g-\frac{\frac{\Omega+\tau}{\tau}+k_{\theta}^{2} \tilde{r} \rho_{i}^{2}\left(\Omega-1-\eta_{i}\right)}{1+\frac{1}{\tau}+\frac{1}{N}\left(\frac{2}{3} \frac{\Omega}{\tau}+\frac{2}{3}-\eta_{i}\right)}\right] \chi=0 .
$$

\section{b. $v_{\| i}=0$ approximation}

From (27), we obtain

$$
\begin{aligned}
& -\left(\frac{1}{q R}\right)^{2} \frac{k_{\theta}^{2} \rho_{i}^{2} v_{A}^{2}}{\omega_{* i}^{2}}\left(\tilde{r}^{\prime} \Psi^{\prime}+\tilde{r} \Psi^{\prime \prime}\right) \\
& \quad-\left[\frac{\Omega+\tau}{\tau}\left(\Omega+\tau \epsilon_{n} g\right)+\eta_{e} \tau \epsilon_{n} g-\frac{\Omega+\tau}{\tau} \frac{\Omega_{1 \Psi}}{\Omega_{1 \Phi}}\right] \Psi=0,
\end{aligned}
$$

and $\Phi=\frac{\Omega_{1} \Psi}{\Omega_{1 \Phi}} \Psi$. 


\section{c. Electrostatic and $v_{\| i}=0$ approximation}

From (28), we get the (local) equation $\Omega_{1 \Phi}=0$, i.e.,

$\frac{\Omega}{\tau}+1+k_{\theta}^{2} \tilde{r} \rho_{i}^{2}\left(\Omega-1-\eta_{i}\right)-\epsilon_{n} g\left(1+\frac{1}{\tau}+\frac{\frac{2}{3} \frac{\Omega}{\tau}+\frac{2}{3}-\eta_{i}}{N}\right)=0$,

which has the solution

$$
\begin{aligned}
\Omega & =-\frac{\delta}{2} \\
& \pm \sqrt{\frac{\delta^{2}}{4}-\frac{\epsilon_{n} g\left[\eta_{i}-\frac{7}{3}+\frac{5}{3} \epsilon_{n} g\left(1+\frac{1}{\tau}\right)+\frac{5}{3} k_{\theta}^{2} \tilde{r} \rho_{i}^{2}\left(1+\eta_{i}\right)\right]}{\frac{1}{\tau}+k_{\theta}^{2} \tilde{r} \rho_{i}^{2}}} \\
\delta & =\frac{1-\epsilon_{n} g\left(1+\frac{10}{3 \tau}\right)-k_{\theta}^{2} \tilde{r} \rho_{i}^{2}\left(1+\eta_{i}+\frac{5}{3} \epsilon_{n} g\right)}{\frac{1}{\tau}+k_{\theta}^{2} \tilde{r} \rho_{i}^{2}} .
\end{aligned}
$$

${ }^{1} \mathrm{~J}$. Weiland, Collective Modes in Inhomogeneous Plasma (Institute of Physics Publishing, 2000).

${ }^{2}$ A. Jarmén, P. Andersson, and J. Weiland, Nucl. Fusion 27, 941 (1987).

${ }^{3}$ P. B. Snyder and G. W. Hammet, Phys. Plasmas 8, 3199 (2001).

${ }^{4}$ J. Nilsson, M. Liljestrőm, and J. Weiland, Phys. Fluids B 2, 2568 (1990).

${ }^{5}$ F. D. Halpern, A. Eriksson, G. Bateman, A. H. Kritz, A. Pankin, C. M. Wolfe, and J. Weiland, Phys. Plasmas 15, 012304 (2008).

${ }^{6}$ A. Ishizawa, Phys. Plasmas 17, 074503 (2010).

${ }^{7}$ A. Casati, C. Bourdelle, X. Garbet, and F. Imbeaux, Phys. Plasmas 15, 042310 (2008).

${ }^{8}$ I. Predebon, C. Angioni, and S. C. Guo, Phys. Plasmas 17, 012304 (2010).

${ }^{9}$ A. Kuley and V. K. Tripathi, Phys. Plasmas 16, 032504 (2009).

${ }^{10}$ A. Jarmén, P. Malinov, and H. Nordman, Plasma Phys. Controlled Fusion 40, 2041 (1998).

${ }^{11}$ S. Guo and J. Weiland, Nucl. Fusion 37, 1095 (1997).
${ }^{12}$ B. Jhowry, J. Anderson, and S. Dastgeer, Phys. Plasmas 11, 5565 (2004).

${ }^{13}$ B. Jhowry and J. Anderson, Phys. Plasmas 10, 782 (2003).

${ }^{14}$ M. J. Pueschel, M. Kammerer, and F. Jenko, Phys. Plasmas 15, 102310 (2008).

${ }^{15}$ M. J. Pueschel and F. Jenko, Phys. Plasmas 17, 062307 (2010).

${ }^{16}$ P. B. Snyder and G. W. Hammet, Phys. Plasmas 8, 744 (2001).

${ }^{17}$ F. Merz and F. Jenko, Nucl. Fusion 50, 054005 (2010).

${ }^{18}$ A. Skyman, H. Nordman, and P. Strand, Phys. Plasmas 19, 032313 (2012).

${ }^{19}$ H. Nordman, A. Skyman, P. Strand, C. Giroud, F. Jenko, F. Merz, V. Naulin, T. Tala, and JET-EFDA Contributors, Plasma Phys. Controlled Fusion 53, 105005 (2011).

${ }^{20}$ J. Anderson, H. Nordman, and J. Weiland, Plasma Phys. Controlled Fusion 42, 545 (2000).

${ }^{21}$ A. Eriksson, H. Nordman, P. Strand, J. Weiland, T. Tala, E. Asp, G. Corrigan, C. Giroud, M. de Greef, I. Jenkins, H. C. M. Knoops, P. Mantica, K. M. Rantamki, P. C. de Vries, K.-D. Zastrow, and JET-EFDA Contributors, Plasma Phys. Controlled Fusion 49, 1931 (2007).

${ }^{22}$ A. Eriksson and J. Weiland, Phys. Plasmas 12, 092509 (2005).

${ }^{23}$ M. Frőjdh and A. Jarmén, Nucl. Fusion 35, 575 (1995).

${ }^{24}$ A. Jarmén and M. Fröjdh, Phys. Fluids B 5, 4015 (1993).

${ }^{25}$ A. Jarmén and P. Malinov, Phys. Plasmas 2, 3055 (1995).

${ }^{26}$ G. W. Hammett and F. W. Perkins, Phys. Rev. Lett. 64, 3019 (1990).

${ }^{27}$ H. Bigliari, P. H. Diamond, and M. N. Rosenbluth, Phys. Fluids B 1, 109 (1989).

${ }^{28}$ R. E. Waltz, R. R. Dominguez, and G. W. Hammett, Phys. Fluids B 4, 3138 (1992).

${ }^{29}$ H. Nordman, A. Jarmén, P. Malinov, and M. Persson, Phys. Plasmas 2, 3440 (1995).

${ }^{30}$ J. Weiland and A. Hirose, Nucl. Fusion 32, 151 (1992).

${ }^{31}$ J. Anderson, J. Li, and Y. Kishimoto, Phys. Plasmas 14, 082313 (2007).

${ }^{32}$ G. M. Steabler, J. E. Kinsey, and R. E. Waltz, Phys. Plasmas 12, 102508 (2005).

${ }^{33}$ J. E. Kinsey, G. M. Steabler, and R. E. Waltz, Phys. Plasmas 15, 055908 (2008).

${ }^{34}$ J. W. Connor, R. J. Hastie, and J. B. Taylor, Phys. Rev. Lett. 40, 396 (1978).

${ }^{35}$ J. Weiland, Phys. Plasmas 11, 3238 (2004).

${ }^{36}$ A. L. Dimits, G. Bateman, M. A. Beer, B. I. Cohen, W. Dorland, G. W. Hammett, C. Kim, J. E. Kinsey, M. Kotschenreuther, A. H. Kritz, L. L. Lao, J. Mandrekas, W. M. Nevins, S. E. Parker, A. J. Redd, D. E. Shumaker, R. Sydora, and J. Weiland, Phys. Plasmas 7, 969 (2000).

${ }^{37}$ A. Hirose and L. Zhang, Phys. Scr. 52, 208 (1995).

${ }^{38}$ A. Hirose, Phys. Fluids B 3, 1125 (1991).

${ }^{39}$ A. Hirose, A. I. Smolyakov, and O. Ishihara, Nucl. Fusion 33, 735 (1993).

${ }^{40}$ A. Hirose and M. Elia, Plasma Phys. Controlled Fusion 37, 853 (1995). 